\title{
Changes of bone metabolism markers following additional isokinetic and isotonic training and its subsequent cessation in advanced level adolescent weightlifters
}

\author{
Mohamad Faiz Abdul Rahim ${ }^{1}$ and Shazlin Shaharudin ${ }^{2, *}$ \\ ${ }^{1}$ School of Medical Sciences, Universiti Sains Malaysia, Kota Bharu, Kelantan, Malaysia; and ${ }^{2}$ School of Health Sciences, \\ Universiti Sains Malaysia, Kota Bharu, Kelantan, Malaysia
}

Copyright: (C) 2018 M. F. Abdul Rahim and S. Shaharudin. This is an open access article licensed under the Creative Commons Attribution License (http://creativecommons.org/licenses/by/4.0/).

\begin{abstract}
Background: Physically active people present overall accelerated bone turnover. However, less is known about the effects of different types of resistance training on bone metabolism markers among adolescent weightlifters. Objective: The purpose of this study was to investigate the effects of isokinetic training versus isotonic training of rotator cuff muscles and one month of cessation of training on blood bone metabolism markers among adolescent advanced level weightlifters. Methods: Sixteen adolescent male weightlifters (age $14.88 \pm 1.31$ years) who represented their state in Malaysia were weight-matched and randomly assigned into two training groups: isokinetic training (FIT, $n=7$ ) and isotonic training (TON, $n=9$ ) groups. The experiment was conducted during the preparatory phase of the training cycle for a total of 24 sessions with three sessions per week for eight weeks using dynamometer or dumbbells. Blood samples were collected before the commencement of training, after training and one month following the cessation of training and analyzed for determining blood metabolism markers of serum osteocalcin, serum C-telopeptide of type 1 collagen (CTX-1), and serum total calcium. Results: Total calcium concentrations were not different between groups at any time, as indicated by a non-significant main effect of time $(F=1.89, p=.166)$ and no condition $\times$ time interaction effect $(F=0.64, p=.533)$. Serum osteocalcin concentrations were not different between groups at any time, as indicated by a non-significant main effect of time $(F=2.10, p=.162)$ and no condition $\times$ time interaction effect $(F=0.024, p=.912)$. Serum CTX-1 concentrations were not different between groups at any time, as indicated by a non-significant main effect of time $(F=3.12, p=.059)$ and no condition $\times$ time interaction effect $(F=1.59$, $p=.221)$. Conclusions: No significant changes were observed in serum total calcium, osteocalcin and CTX-1 following either eight weeks of isokinetic or isotonic additional training among advanced level of adolescent weightlifters.
\end{abstract}

Keywords: bone resorption, bone absorption, resistance training, weight training

\section{Introduction}

Bone metabolism can be divided into two processes which is bone formation and bone resorption. The rate of these processes can be determined by urine or serum biochemical markers which indirectly reflect bone metabolism. Bone formation markers include serum osteocalcin while bone resorption markers include serum C-terminal telopeptide of type 1 collagen (CTX-1; Maïmoun \& Sultan, 2011). Bone metabolism can be influenced by many types of exercise. Bone loading through exercise during adolescent provides a

\footnotetext{
* Address for correspondence: Shazlin Shaharudin, Exercise \& Sports Science Programme, School of Health Sciences, Universiti Sains Malaysia, 16150 Kota Bharu, Kelantan, Malaysia. E-mail: shazlin@usm.my
}

"window of opportunity" for healthy bone development (Forwood, 2013). However, the exercise effects on bone turnover markers in the skeleton of growing athletes have received little attention (Maïmoun \& Sultan, 2011).

Typical weightlifting training involves isotonic muscle contraction of lifting a fixed weight such as dumbbell and barbell. Examples of isotonic training are biceps and triceps curl. In isotonic contraction, the resistance remains constant throughout the range of motion of the involved joint (Kovaleski, Heitman, Trundle, \& Gilley, 1995; Smith \& Melton, 1981). Therefore, loading occurs at the weakest point in the system, while the rest of the system is functioning at less than its capacity (Smith \& Melton, 1981). On the other hand, isokinetic training mode provides proportional resistance following the amount of force that being exerted. This allows a constant angular velocity 
across the whole range of motion of the involved joint (Kraemer \& Ratamess, 2004). Therefore, a maximal effort can be experienced during isokinetic contraction because the maximal load is applied throughout the range of motion. Previously, significant increase in dynamic and static strength in both isokinetic and isotonic groups was observed following eight weeks of standardized training programs of the knee extensors (Remaud, Cornu, \& Guével, 2010). Hence, the authors concluded that the two training methods could be proposed to enhance concentric muscle strength in dynamic and static conditions. However, to the best of our knowledge, comparison on the effects of these two training methods was not yet studied in terms of bone marker metabolism.

Isokinetic resistance training is osteogenic, whereby it can initiate new bone tissue (Nickols-Richardson, Miller, Wootten, Ramp, \& Herbert, 2007). This is due to the constant force applied on the muscle through the entire range of motion (Balnave \& Thompson, 1993; Hather, Tesch, Buchanan, \& Dudley, 1991; Higbie, Cureton, Warren, \& Prior, 1996), which is an advantage compared to the isotonic type of training. In a study conducted by Miller et al. (2006), it was found that high intensity and low velocity of both eccentric and concentric isokinetic training improved total body bone mineral content and site-specific bone mineral density and bone mineral content among young women. In another study conducted by Miller et al. (2007) it was observed that isokinetic strength training could enhance bone stiffness as well as bone mineral content and bone mineral density. Regarding effects of isokinetic resistance exercises or training on bone metabolism markers, a previous study by Schroeder, Hawkins, and Jaque (2004) reported increased of serum osteocalcin, a bone formation marker, following 16 weeks of isokinetic resistance training among young women. On the other hand, it was reported that power lifters of age 19-24 years old who underwent high intensity isotonic resistance training for 12 months had a higher bone mineral density in the lumbar spine compared to control group (Tsuzuku, Ikegami, \& Yabe, 1998). Moreover, it was shown previously that isotonic resistance exercise training increased bone formation markers and it transiently suppressed the bone resorption marker (Fujimura et al., 1997).

Physically active people present overall accelerated bone turnover (Maïmoun \& Sultan, 2011). However, new steady state in bone turnover was achieved after a few weeks of reduced activity that would partly explain the faster bone mineral density loss observed in former athletes (Bass et al., 1998). For example, within four weeks of reduced activity during the recovery period between two sports seasons, a decrease in concentration of bone formation markers and an increase in concentration of bone resorption markers among soccer players were observed (K. M. Karlsson et al., 2003).

However, to our knowledge, studies that compare the effects of types of resistance training (isotonic versus isokinetic) are scarce, particularly in advanced levels of adolescent athletes. Therefore, the aim of this study was to investigate the effects of eight weeks of either isokinetic or isotonic training and its subsequent four weeks of cessation of training on blood bone metabolism markers among advanced levels of adolescent male weightlifters. We hypothesized that both types of training may enhance bone formation markers during training phase and reduce the markers' concentration following training cessation.

\section{Methods}

\section{Participants}

Adolescent (13-17 years old) male weightlifters with experience in competitive weightlifting (i.e., representing their state) and without previous history of shoulder injury were included. Participants were excluded from the study if they were unable to adhere to at least $85 \%$ of training loads during the intervention and/or had undergone rehabilitation for any musculoskeletal injury within the last two years.

At the pre-test, 20 male adolescent weightlifters volunteered to participate in the study. Participants were provided with a detailed explanation regarding the methodology of the study. Upon agreement, their written consent and assent from their guardians were obtained. The participation in the present study was voluntary. Study procedures were conducted in compliance to Declaration of Helsinki and the ethical approval was obtained from Human Ethical Committee of a local university (USM/JEPeM/14110457). Then, participants were weight-matched and randomly assigned to isokinetic (FIT, $n=10$ ) or isotonic (TON, $n=10)$ groups.

Anthropometry measurement was taken before and after the experimental period, and one month after cessation of training. Body mass, percentage of body fat and fat free mass were measured using a body composition analyzer (TANITA, Tokyo, Japan). Participants' body height was measured using a body height scale (SECA, Hamburg, Germany).

\section{Experimental design}

The study procedures were conducted during the preparatory phase of the weightlifters' training cycle. All participants were recruited from the same pool of weightlifters with standardized training program 
provided by their coach. Their program included five strength training sessions and one tactical session per week. The duration of their standardized training session was two hours per session. Meanwhile, the training program prescribed in the current study (isokinetic and isotonic upper limb resistance training) was an additional training for the athletes.

The TON group had their training at a state gymnasium while the FIT group had their isokinetic training at a sport science laboratory of a local university. The experiment was conducted for a total of 24 sessions within a period of eight weeks (three sessions per week). Blood samples were taken and analyzed for bone metabolism markers (total serum calcium, serum osteocalcin, serum CTX-1) the commencement of training program, immediately after the completion of training program and one month following the training cessation.

\section{Study procedure}

Isokinetic and isotonic training program

The isokinetic training was applied using an isokinetic dynamometer (Biodex Pro, Shirley, NY, USA). The dynamometer was calibrated with the known weight before each training sessions. A correction for the mass of the limb and lever arm system was made on all torque curves. The preparation of the participant on the dynamometer followed the manufacturer's manual closely to ensure safety of the participants.

For each training sessions, there was ten minutes of warming up, emphasizing the shoulder joint which was performed by each participant followed by a minute of active rest. For cool down, the participants stretched their shoulder. The details of the program have been summarized in Table 1. Each training session took approximately one hour including the warm up and stretching. Progressive loads in terms of angular velocity, position of the shoulder joint, number of sets and repetitions were applied in the eight-weeks training program.

The same lifting positions, duration of rest interval between sets, number of sets and repetitions were applied by the isotonic group however their training was conducted using a constant weight (dumbbells) of $50 \%$ of their upper limb's weight and the angular velocity was not fixed in isotonic group (Abdul Rahim, Wan Mohamed, \& Shaharudin, 2017; Wan Mohamed, Abdul Rahim, \& Shaharudin, 2017). The participants' upper limb weight was measured using an isokinetic device before the commencement of the training program.

\section{Blood sample collection and handling}

Seven $\mathrm{ml}$ of blood was drawn from each participant by a trained laboratory technologist at the sport science laboratory. Blood samples were collected at 8.30 a.m. ( \pm 30 minutes) after an overnight fast (water consumption was allowed; Shaharudin, Abdul Rahim, Muhamad, 2018). Samples were centrifuged using centrifuge machine (Hettich-Rotina, Tuttlingen, Germany) for 10 minutes at $4000 \mathrm{rpm}$ in $4{ }^{\circ} \mathrm{C}$ temperature to obtain serum. Next, the serum was divided into equal portions and stored in a freezer (ThermoForma, Midland, ON, Canada) at $-80{ }^{\circ} \mathrm{C}$ for further analysis.

\section{Bone metabolism marker analysis}

Serum total calcium was analyzed using Calcium Colorimetric Assay Kit (Sigma-Aldrich, Darmstadt, Germany) and measured colorimetrically by using Roche-Hitachi 912 Chemistry Analyser (Hitachi, Tokyo, Japan). Enzyme immunological test kit (N-MID ${ }^{\circledR}$ Osteocalcin ELISA, Immunodiagnostic Systems Holdings, East Boldon, United Kingdom) was used to analyze serum osteocalcin and the concentration was determined using a photometric microplate reader (Molecular Devices, San Jose, CA, USA). Serum CTX-1 (bone resorption marker) concentration was analyzed using Human CTX-1 ELISA kit (QayeeBio, Shanghai, China). Then, the concentration was measured using Versa ${ }_{\text {Max }}$ ELISA Microplate Reader (Molecular Devices, San Jose, CA, USA).

\section{Statistical analysis}

A priori sample size calculation showed that eight participants per group are sufficient to yield 0.8 power of the study with effect size of 0.6 (large sample size according to Cohen, 1988). Descriptive data were expressed as mean \pm standard deviation. The physical

Table 1

Details of additional isokinetic and isotonic training programs

\begin{tabular}{|c|c|c|c|c|c|}
\hline Session & Body position & Repetitions & Sets & Rest (min) & Velocity $\left({ }^{\circ} \cdot \mathrm{s}^{-1}\right)$ \\
\hline 1 to 8 & Seated with $45^{\circ}$ of shoulder abduction & $12-15$ & 2 & 1 & 120 \\
\hline 9 to 16 & Seated with $90^{\circ}$ of shoulder abduction & $10-12$ & 3 & 1 & 240 \\
\hline 17 to 24 & Standing while lifting the bar diagonally overhead & $8-10$ & 4 & 1 & 360 \\
\hline
\end{tabular}

Note. Similar body positions, repetitions, sets and rest intervals were used for both training programs. 
characteristics of participants were compared using independent $t$-test. The differences between pre- and post-tests and between after training cessation and post-test for each calcium, osteocalcin and CTX-1 concentration were expressed in percentage. The normality of data distribution was confirmed using Shapiro-Wilk normality test. A general linear model two-way analysis of variance (ANOVA) with repeated measure design was used for statistical analysis in which the within subjects factor was time (before training, immediately after training and subsequent four weeks after cessation of training) and the between subjects factor was group (FIT and TON). Significant main or interaction effects were followed by post hoc analyses with a Bonferroni correction. The accepted level of significance was set at $\alpha=.05$. Data were analyzed using SPSS (Version 22; IBM, Armonk, NY, USA).

\section{Results}

Four of the participants dropped out from the study due to lack of adherence (less than $85 \%$ ) to the additional training program. Hence, statistical analysis was conducted on the data of the remaining 16 participants $(\mathrm{FIT}=7, \mathrm{TON}=9)$. Participants' physical characteristics are presented in Table 2. Before the training period, there were no statistically significant differences among the groups on the physical characteristics of participants.

The results of blood metabolism markers are presented in Table 3. Total calcium concentrations were not significantly different between groups at any time point, as indicated by a non-significant main effect of time $(F=1.89, p=.166)$ and no condition $\times$ time interaction effect $(F=0.64, p=.533)$. Serum osteocalcin concentrations were not different between groups at any time point, as indicated by a non-significant main effect of time $(F=2.10, p=.162)$ and no condition $\times$ time interaction effect $(F=0.024, p=.912)$. Similarly, serum CTX-1 concentrations were not different between groups at any time point, as indicated by a non-significant main effect of time $(F=3.12$, $p=.059)$ and no condition $\times$ time interaction effect $(F=1.59, p=.221)$.

Table 2

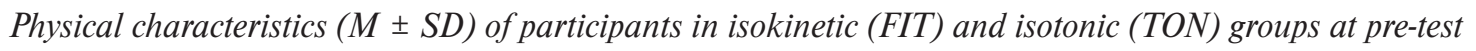

\begin{tabular}{lccc}
\hline Variable & FIT $(n=7)$ & TON $(n=9)$ & $p$ \\
\hline Age (years) & $14.43 \pm 0.98$ & $15.22 \pm 1.48$ & .242 \\
Experience (months) & $13.29 \pm 8.12$ & $21.11 \pm 11.44$ & .148 \\
Body mass (kg) & $70.78 \pm 21.20$ & $59.07 \pm 10.88$ & .171 \\
Height (m) & $1.59 \pm 0.07$ & $1.60 \pm 0.11$ & .977 \\
Fat-free mass (kg) & $46.19 \pm 9.04$ & $49.46 \pm 15.79$ & .634 \\
Body fat (\%) & $32.50 \pm 11.46$ & $24.08 \pm 6.10$ & .078 \\
\hline
\end{tabular}

Table 3

Serum total calcium, osteocalcin and C-telopeptide of type 1 collagen (CTX-1) before (pre-test), after eight weeks of training (post-test) and after one month of cessation of training in isokinetic (FIT) and isotonic (TON) groups. Values are presented as $M \pm S D$.

\begin{tabular}{|c|c|c|c|c|c|}
\hline & Pre-test & Post-test & $\begin{array}{l}\text { After one month } \\
\text { of cessation of } \\
\text { training }\end{array}$ & $\begin{array}{c}\% \text { of difference } \\
\text { between pre- and } \\
\text { post-test }\end{array}$ & $\begin{array}{c}\% \text { of difference } \\
\text { between pre-test } \\
\text { and after one } \\
\text { month of cessation } \\
\text { of training }\end{array}$ \\
\hline \multicolumn{6}{|l|}{ FIT $(n=7)$} \\
\hline Calcium $\left(\mathrm{mmol} \cdot \mathrm{L}^{-1}\right)$ & $2.37 \pm 0.07$ & $2.34 \pm 0.05$ & $2.36 \pm 0.07$ & -1.27 & -0.42 \\
\hline Osteocalcin $\left(\mathrm{ng} \cdot \mathrm{ml}^{-1}\right)$ & $79.28 \pm 21.70$ & $84.62 \pm 11.82$ & $78.03 \pm 8.72$ & +6.74 & -1.58 \\
\hline CTX-1 (ng $\left.\cdot \mathrm{ml}^{-1}\right)$ & $59.83 \pm 4.87$ & $60.93 \pm 6.41$ & $57.28 \pm 9.59$ & +1.84 & -4.26 \\
\hline \multicolumn{6}{|l|}{ TON $(n=9)$} \\
\hline Calcium $\left(\mathrm{mmol} \cdot \mathrm{L}^{-1}\right)$ & $2.39 \pm 0.05$ & $2.37 \pm 0.07$ & $2.35 \pm 0.08$ & -0.83 & -1.67 \\
\hline Osteocalcin $\left(\mathrm{ng} \cdot \mathrm{ml}^{-1}\right)$ & $67.17 \pm 22.72$ & $69.91 \pm 16.19$ & $67.90 \pm 16.81$ & +4.08 & +1.09 \\
\hline CTX-1 (ng $\left.\cdot \mathrm{ml}^{-1}\right)$ & $55.06 \pm 7.68$ & $59.01 \pm 8.30$ & $55.42 \pm 9.09$ & +7.17 & +0.65 \\
\hline
\end{tabular}




\section{Discussion}

In the present study, eight weeks of either FIT or TON training (24 sessions in total) did not significantly affect any markers of bone metabolism. However, both types of training decreased serum osteocalcin and increase CTX-1 although these changes were not statistically significant. A previous study by Bell, Godsen, Henry, Shary, and Epstein (1988) reported that high levels of osteocalcin were observed in young men participating in regular muscle building activities and weightlifting (M. K. Karlsson, Vergnaud, Delmas, \& Obrant, 1995). Meanwhile, Schroeder et al. (2004) conducted a research on the musculoskeletal adaptation to 16 weeks of isotonic eccentric progressive resistance training among young women aged $18-28$ years. The authors reported that the level of serum osteocalcin dropped in the first eight weeks of intervention but increased until the sixteenth week for both high intensity training and low intensity training. Consistent with their findings, the present study also found that there was a trend of serum osteocalcin level decreased after eight weeks of study period, even though it was not significant. However, in the present study, the intervention period was only eight weeks, therefore we speculated bone adaptation may need a longer period of time to response to the resistance training as the average turnover at bone remodelling site of the bone may take several months as reported in Recker et al. (1988). Inconsistent with the present study, Fujimura et al. (1997) reported an increase of serum osteocalcin within the first month of resistance exercise which remained constant throughout the intervention period. However, they did not observe change in plasma procollagen type-I C-terminal (PICP) concentration throughout the intervention. This might be due to increased rates of bone formation which transiently suppressed the bone resorption process.

Type 1 collagen fibers are insoluble in nature and constitute of $90 \%$ of the organic matrix of the bone architecture and their degradation is important for bone resorption process. The responsible cell for bone resorption is osteoclast. As a result of the degradation, several collagen fragments are released into the blood circulation. The components that contribute most from the bone include CTX-1 and cross-linked carboxyterminal telopeptide of type 1 collagen (1CTP; Seibel, 2005). CTX fragments is highly bone specific as osteoclasts cells are not activated during the degradation of other than type-1 collagen fibers (Christenson, 1997) and therefore appears to be sensitive markers to detect the process of bone resorption.

Regarding isokinetic training and bone health status, Miller et al. (2007) reported an increase of bone mineral content and bone mineral density after 20 weeks of legs and arm isokinetic training among young women. This reflects the process of bone turnover after isokinetic training. This is due to the constant force applied by muscle contraction on bones through the entire range of motion during isokinetic resistance training (Balnave \& Thompson, 1993; Higbie et al., 1996). Unfortunately, we did not measure bone health status in the present study, therefore, results on bone mineral content and density are unable to be reported and this is a limitation of the current study.

Regarding effects of cessation of training, it was observed that four weeks of cessation of training did not significantly affect all the measured blood parameters in both FIT and TON groups. It should be noted that the mandatory training was on-going during the cessation period of our additional training program. It is a common situation in studying competitive athletes whereby it is not possible to exclude them from any form of training. However, measures were taken to ensure that no drastic change in their mandatory training program was carried out during the cessation period of the additional training program. Our findings concur with Sartorio et al. (2006), whereby no significant variation in bone marker values were observed over six months of a sporting season which entails periods of training, competition, recovery, and resting in athletes from different sporting disciplines. In a review paper by Maïmoun and Sultan (2011), highly trained subjects seem to have relatively stable bone remodeling during the sport season, which may explain the lack of significant changes in our results. On the contrary, soccer players showed a decrease in bone formation and an increase in bone resorption within four weeks of reduced activity during the recovery period between two sports seasons (K. M. Karlsson et al., 2003).

In conclusion, both types of resistance training (isokinetic and isotonic) could increase bone resorption marker osteocalcin and bone formation marker CTX-1. However, since bone turnover is a dynamic process it might fluctuates to give way for other process to occur which would be either resorption or formation. In order for the process of bone formation to occur, bone resorption must take place. Therefore, the equilibrium would highly depend on the stress places on the bones from contraction of skeletal muscles.

\section{Limitations}

The additional training program involved only the shoulder joint. However, bone formation and resorption markers represent an average of the turnover in all skeletal sites and, thus, is not site specific. During the cessation period, only the additional training program was attenuated, however participants were still 
involved in their mandatory training program. Initially, 20 male adolescent weightlifters were recruited. They were weight-matched and randomly assigned to either isokinetic or isotonic group. However, four subjects dropped out from the study (three from FIT group and one from TON group) due to inability to complete the additional training program following participation in international competition. The subjects who dropped out are experienced weightlifters with approximately three years of experience in competitive weightlifting. Their absence caused insignificant changes in terms of subjects' physical characteristics across groups, which have been matched prior to the training program.

\section{Conclusion}

Eight weeks of resistance training of isokinetic and isotonic training elicited similar findings on serum total calcium, osteocalcin and CTX-1. In addition, these two types of training may have potential in reducing bone resorption with four weeks cessation of training among male adolescent weightlifters.

\section{Acknowledgment}

The study was funded by MOHE Fundamental Research Grant Scheme (203/PPSP/6171165) and Universiti Sains Malaysia (304/PPSP/61313152).

\section{Conflict of interest}

There were no conflicts of interest.

\section{References}

Abdul Rahim, M. F., Wan Mohamed, I., \& Shaharudin, S. (2017). Effects of isokinetic versus isotonic training of shoulder joint on 2D kinematics and barbell velocity during power clean and power snatch of advanced level of adolescent weightlifters. International Journal of Sports Science, 7, 144-150.

Balnave, C. D., \& Thompson, M. W. (1993). Effect of training on eccentric exercise-induced muscle damage. Journal of Applied Physiology, 55, 1545-1551.

Bass, S., Pearce, G., Bradney, M., Hendrich, E., Delmas, P. D., \& Harding, A. (1998). Exercise before puberty may confer residual benefits in bone density in adulthood: Studies in active prepubertal and retired female gymnasts. Journal of Bone and Mineral Research, 13, 500-507.

Bell, N. H., Godsen, R. N., Henry, D. P., Shary, J., \& Epstein, S. (1988). The effects of muscle-building exercise on vitamin D and mineral metabolism. Journal of Bone and Mineral Research, 3, 369-373.

Christenson, R. H. (1997). Biochemical markers of bone metabolism: An overview. Clinical Biochemistry, 30, 573-593.

Cohen, J. (1988). Statistical power analysis for the behavioral sciences (2nd ed.). Hillsdale, NJ: Lawrence Erlbaum Associates.

Fujimura, R., Ashizawa, N., Watanabe, M., Mukai, N., Fukubayashi, T., Hayashi, K., ... Suzuki, M. (1997). Effect of resistance exercise training on bone formation and resorption in young male subjects assessed by biomarkers of bone metabolism. Journal of Bone and Mineral Research, 12, 656-662.

Forwood, M. R. (2013). Growing a healthy skeleton: The importance of mechanical loading. In C. J. Rosen, R. Bouillon, J. E. Compston, \& V. Rosen (Eds.), Primer on the metabolic diseases and disorders of mineral metabolism (pp. 149-155). Hoboken, NJ: John Wiley \& Sons.

Hather, B. M., Tesch, P. A., Buchanan, P., \& Dudley, G. A. (1991). Influence of eccentric actions on skeletal muscle adaptations to resistance training. Acta Physiologica Scandinavica, 143, 177-185.

Higbie, E. J., Cureton, K. J., Warren, G. L., III, \& Prior, B. M. (1996). Effects of concentric and eccentric training on muscle strength, cross-sectional area, and neural activation. Journal of Applied Physiology, 81, 2173-2181.

Karlsson, K. M., Karlsson, C., Ahlborg, H. G., Valdimarsson, O., Ljunghall, S., \& Obrant, K. J. (2003). Bone turnover responses to changed physical activity. Calcified Tissue International, 72, 675-680.

Karlsson, M. K., Vergnaud, P., Delmas, P. D., \& Obrant, K. J. (1995). Indicators of bone formation in weight lifters. Calcified Tissue International, 56, 177-180.

Kovaleski, J. E., Heitman, R. H., Trundle, T. L., \& Gilley, W. F. (1995). Isotonic preload versus isokinetic knee extension resistance training. Medicine \& Science in Sports \& Exercise, 27, 895-899.

Kraemer, W. J., \& Ratamess, N. A. (2004). Fundamentals of resistance training: Progression and exercise prescription. Medicine \& Science in Sports \& Exercise, 36, 674-688.

Maïmoun, L., \& Sultan, C. (2011). Effects of physical activity on bone remodelling. Metabolism Clinical and Experimental, 60, 373-388.

Miller, L. E., Pierson, L. M., Nickols-Richardson, S. M., Wootten, D. F., Selmon, S. E., Ramp, W. K., \& Herbert, W. G. (2006). Knee extensor and flexor torque development with concentric and eccentric isokinetic training. Research Quarterly for Exercise and Sport, 77, 58-63.

Miller, L. E., Wootten, D. F., Nickols-Richardson, S. M., Ramp, W. K., Steele, C. R., Cotton, J. R., ... Herbert, W. G. (2007). Isokinetic training increases ulnar bending stiffness and bone mineral in young women. Bone, 41, 685-689.

Nickols-Richardson, S. M., Miller, L. E., Wootten, D. F., Ramp, W. K., \& Herbert, W. G. (2007). Concentric and eccentric isokinetic resistance training similarly increases muscular strength, fat-free soft tissue mass, and specific 
bone mineral measurements in young women. Osteoporosis International, 18, 789-796.

Recker, R. R., Kimmel, D. B., Parfitt, A. M., Davies, K. M., Keshawarz, N., \& Hinders, S. (1988). Static and tetracycline-based bone histomorphometric data from 34 normal postmenopausal females. Journal of Bone and Mineral Research, 3, 133-144.

Remaud, A., Cornu, C., \& Guével, A. (2010). Neuromuscular adaptations to 8-week strength training: Isotonic versus isokinetic mode. European Journal of Applied Physiology, 108, 59-69.

Sartorio, A., Jubeau, M., Agosti, F., Marazzi, N., Rigamonti, A., Muller, E. E., \& Maffiuletti, N. A. (2006). A follow-up of GH-dependent biomarkers during a 6-month period of the sporting season of male and female athletes. Journal of Endocrinological Investigation, 29, 237-243.

Schroeder, E. T., Hawkins, S. A., \& Jaque, S. V. (2004). Musculoskeletal adaptations to 16 weeks of eccentric progressive resistance training in young women. Journal of Strength and Conditioning Research, 18, 227-235.
Seibel, M. J. (2005). Biochemical markers of bone turnover part I: Biochemistry and variability. Clinical Biochemist Reviews, 26, 97-122.

Shaharudin, S., Abdul Rahim, M. F., \& Muhamad, A. S. (2018). Effects of isokinetic versus isotonic training and its cessation on total leukocytes and lymphocytes count in adolescent state-level weightlifters. International Journal of Preventive Medicine, 9, 90.

Smith, M. J., \& Melton, P. (1981). Isokinetic versus isotonic variable resistance training. American Journal of Sports Medicine, 9, 275-279.

Tsuzuku, S., Ikegami, Y., \& Yabe, K. (1998). Effects of highintensity resistance training on bone mineral density in young male powerlifters. Calcified Tissue International, 63, 283-286.

Wan Mohamed, I., Abdul Rahim, M. F., \& Shaharudin, S. (2017). Effects of isokinetic versus isotonic training on strength, power and muscular balance of rotator cuff muscles among advanced level of adolescent weightlifters. International Journal of Applied Sports Science, 29, 143-154. 